\title{
Caracterización de las competencias digitales en estudiantes universitarios de Chiclayo a raíz de la covid 19
}

\author{
Susana Angelica Dávila Guevara \\ suandague@gmail.com \\ Orcid: 0000-0003-2676-137X \\ Estudiante de doctorado de la escuela de postgrado \\ Universidad Cesar Vallejo UCV \\ Chiclayo -Perú
}

\section{RESUMEN}

Este artículo presenta los resultados de un proceso de investigación con estudiantes del primer ciclo de la Universidad Tecnológica del Perú, el cual tiene como objetivo evidenciar el desarrollo de las competencias digitales, específicamente lo relacionado con el manejo de información durante sus clases virtuales, para lo cual se plantea una metodología cuantitativa de tipo descriptivo analítico con un diseño no experimental. Como instrumento se aplicó un cuestionario, en donde se evidenció que los estudiantes acceden constantemente a internet, sin embargo, pocos conocen sobre las competencias digitales, debido a que interactúan constantemente en redes sociales, por lo cual dejan de lado las actividades académicas.

Palabras claves: Competencia digital, proceso de enseñanza - aprendizaje, educación superior, manejo de información, tecnología. 


\title{
Characterization of digital skills in Chiclayo university students as a result of covid 19
}

\begin{abstract}
The following article presents the results of a research project among first cycle students of the Technological University of Peru which has as the objective evince the digital competence development, specifically what is related to the information management during online classes for which a quantitative methodology with an analytical descriptive method and a nonexperimental design is proposed. The instrument applied was a questionnaire where it was shown that students access constantly to the Internet; however, only a few are aware of the digital competences due to their constant interaction in social networks, which is the main reason students let the academic activities aside.
\end{abstract}

Keywords: Digital competence, teaching-learning process, higher education, information management, technology.

Artículo recibido: 05 de Mayo 2021 Aceptado para publicación: 20 de Junio 2021 Correspondencia: suandague@gmail.com Conflictos de Interés: Ninguna que declarar 


\section{INTRODUCCIÓN}

Actualmente el mundo vive uno de sus peores momentos a causa de la pandemia de Covid-19, producida por una cepa mutante de coronavirus el SARS-CoV-2. Esta pandemia se inició en China a finales del 2019 y posteriormente se extendió en todo el mundo. La Organización Mundial de la Salud (OMS) declaró al covid-19 como una nueva pandemia mundial (Maguiña, Acosta y Tequen, 2020).

El cuadro clínico característico de la covid-19 se inicia con fiebre, tos seca y disnea, sin embargo, en algunos casos se pueden experimentar otros síntomas más fuertes que pueden provocar la muerte. Estas complicaciones son frecuentes en las personas con enfermedades crónicas (diabetes mellitus, hipertensión arterial, asma, obesidad, etc.). Tal ha sido la expansión de la enfermedad que 1,7 millones de personas han fallecido a causa de este virus (Córdova y Rossani, 2020).

En el Perú, el SARS-CoV-2 se registró con el paciente 0 a mediados de marzo del 2020 y debido a la eminente proliferación de contagios en la población, el gobierno estableció medidas de cuarentena y distanciamiento social, lo cual tuvo un impacto negativo en diversos sectores, tales como salud, economía y educación.

Ante este contexto de la emergencia sanitaria, las personas se han visto en la necesidad de permanecer confinados en casa. Por lo cual, cada vez más personas trabajan desde casa, sin embargo, en el tema educativo, aún existen muchas deficiencias, debido a que hay alumnos que no tienen acceso a internet, a un televisor o una radio, por lo cual la educación a distancia se convierte para muchos en un imposible. Según datos brindados por la UNESCO, más de 861.7 millones de niños y jóvenes en 119 países se han visto afectados en su educación a causa de la covid-19 (Murillo y Duk, 2020).

En un escenario educativo se está acostumbrado a planificar las actividades que se desarrollarán a lo largo de un semestre, ciclo o año escolar, sin embargo, de un momento a otro los docentes se vieron obligados a mudarse al entorno digital, un escenario con el cual muchas veces no se familiarizan. A pesar de las herramientas tecnológicas que contribuyen con la educación, muchos docentes no están los suficientes preparados para asumir este reto y a tomar estrategias adecuadas en clase. No obstante, es necesario que instituciones, docentes, alumnos y familia estén dispuestos a adaptarse y a tomar aspectos útiles de ésta, teniendo una aproximación y una apropiación de lo que es (Mendoza, 2020). 
Las escuelas de educación básica iniciaron el año escolar en marzo, sin embargo, se vieron en la necesidad de frenar sus actividades por disposiciones del gobierno, teniendo la expectativa de retomar a mediados de abril, sin imaginar lo que pasaría después con el foco fuerte de contagio del virus, lo cual hizo inevitable el regreso a clases. Con respecto a la educación superior tuvieron que trasladarse al entorno digital, asumiendo una modalidad emergente a distancia. Sin embargo, el problema se presentó en las confusiones, equívocos y tensiones que se han generado durante las clases virtuales, una situación que algunos docentes han sabido superar debido a su buena actitud y empatía con sus estudiantes, quienes fueron los actores clave en la transferencia emergente de la modalidad presencial a lo que ahora se conoce como Enseñanza Remota (Silas y Vázquez, 2020).

Por lo tanto, el problema radica en que muchas veces el docente no tiene conocimiento sobre las competencias digitales, para así desarrollar saberes o habilidades que les permitan estar en mejores condiciones para dar respuesta a las necesidades de enseñanza que los modelos mixtos, síncronos y asíncronos están demandando actualmente. Lo cual es preocupante ya que muchos docentes no tienen la suficiente capacitación para enseñar sus contenidos de manera virtual y potenciar las competencias digitales (Aguirre y Ruiz, 2016). "La competencia digital; es una competencia clave e implica seguridad en el uso de las tecnologías de información social, en contextos de entretenimiento y comunicación, apoyándose de las distintas herramientas tecnológicas; y la valoración de la información disponible” (Rojas, Zeta y Jiménez, 2020. p.16).

Los estudiantes del siglo XXI están vinculados con la tecnología, se pasan varias horas del día interactuando y navegando por internet, por lo cual los docentes deben requerir de capacidades que faciliten a los estudiantes adaptarse rápidamente a los cambios que implica las clases remotas en un entorno virtual. Los estudiantes deben tener conocimiento sobre aplicaciones, estrategias de búsqueda por internet y tratamiento de la información manteniendo una actitud crítica, reflexiva, responsable y ética (Chávez, Cantú y Rodríguez, 2016).

Para hacer una clase virtual primero se necesita un campus virtual o plataforma como Blackboard, Microsoft Teams, Moodle, Zoom o Collaborate, y segundo que el profesor sepa cómo funciona y esté en condiciones de usarlo. No obstante, el aprendizaje en el entorno virtual suele ser algo complejo. Durante este proceso los estudiantes, deben 
mantener una comunicación constante y coordinar las tareas que se deben de realizar (Caraballo, Domínguez y Paralela, 2011).

Por otro lado, el reto que asume la educación superior es adaptar las clases virtuales a las necesidades de los estudiantes. Las aulas de clases quedaron desplazadas por las casas de los alumnos, y uno de los retos más importantes ha sido encontrar espacios para separar la vida académica y la de ocio. Ahora bien, adoptando una perspectiva diferente, se ha evidenciado un déficit en las herramientas digitales por parte de los estudiantes. A pesar que más de la mitad de ellos, pasa de más de cinco horas diarias conectados a la red ya se a través de la PC, computador portátil, Tablet o smartphones de última generación, no son capaces de extraer un mayor aprovechamiento de dichas plataformas y por ende se percibe cierta obsolescencia de competencias digitales, ya que utilizan la tecnología para jugar, interactuar en redes sociales y realizar otras actividades alejadas del proceso educativo (Levano, Sánchez, Guillén, Tello, Herrera y Collantes, 2019).

Las denominadas competencias digitales son entendidas a manera de concepto que ha generado diversas líneas de investigación que a luz de los nuevos avances tecnológicos en el rubro de las TICs. Su vasta trascendencia en el ámbito de su aplicación a la Tecnología Educativa, cuyo espectro de acción abarca diversas proyecciones, tanto de aprendizajes, investigación, recreacionales y sociales, entre otros. (Levano Francia, et.al, 2019, p.25)

La competencia digital es el conjunto de conocimientos, habilidades, actitudes, estrategias y concienciación que el uso de las TIC y de los medios digitales requiere para realizar tareas, resolver problemas, comunicar, gestionar la información, colaborar, crear y compartir contenidos y generar conocimiento de forma efectiva, eficaz, adecuada, crítica, creativa, autónoma, flexible, ética, reflexiva para el trabajo, el ocio, la participación, el aprendizaje, la socialización, el consumo y el empoderamiento. Entre las principales competencias digitales tenemos: la alfabetización en información y datos, la comunicación, la creación de contenidos digitales, seguridad y resolución de problemas (Padilla, Gámiz y Romero, 2019).

En tal sentido, las competencias digitales representan el motor de desarrollo de la educación en América Latina. Tomando en cuenta el informe de la Comisión Económica para América Latina y el Caribe (CEPAL) se muestra que el porcentaje de docentes que 
ha tomado cursos sobre TIC es bastante alto en países como: Chile (90\%), Perú (82\%), Colombia (61\%) o Costa Rica (60\%), mientras el porcentaje de docentes que ha tomado cursos sobre TIC es bastante bajo en países en vías de desarrollo como: Nicaragua (17\%), Paraguay (10\%) o Guatemala (6\%), lo cual es algo preocupante (Cobos, Jaramillo y Vinueza, 2019).

En este esquema de utilización de las TIC no sólo los estudiantes tienen un papel importante; al docente le corresponde dar un sentido pedagógico a las herramientas digitales, con el trabajo reflexivo y colaborativo con sus compañeros. También los padres de familia tienen la oportunidad de fortalecer el vínculo escuela-hogar y motivar a los niños en su desarrollo personal (Cabero y Llorente, 2008)

Las universidades tienen que tomar la iniciativa de incluir las TIC y las herramientas Web 2.0 y 3.0 dentro del currículo educativo. De esta manera los estudiantes podrán adquirir las competencias digitales necesarias para trabajar en una sociedad completamente digital o bien, desarrollar sus habilidades tecnológicas en un entorno digital. (Arias, Torres y Yañez, 2014, p. 363)

Según Aguirre y Ruiz (2012) la educación virtual está creciendo considerablemente en México, principalmente en la educación superior, que no solo han ampliado la oferta educativa, sino que además la diversifican. Esto ha generado que las diversas universidades y su staff de docentes se vean en la obligación de capacitarse en competencias digitales, para así desarrollar habilidades que les permitan dar respuesta a las nuevas necesidades de enseñanza que los modelos mixtos, síncronos y asíncronos están demandando actualmente.

Es importante indicar que cada día millones de peruanos se conectan a Internet para compartir ideas, conversar con amigos, realizar trámites y llevar sus clases virtuales. En este escenario, la educación peruana tiene que preparar a los estudiantes en el desarrollo de competencias digitales que les permita trabajar en equipo o aprender autónomamente. En este escenario, se debe formar y empoderar a los docentes en prácticas pedagógicas enfocadas en contextos digitales (Levano, et.al, 2019). (Levano Francia, y otros, 2019) La Ley Universitaria peruana, Ley $\mathrm{N}^{\circ} 30220$ declara que "la universidad es una comunidad académica orientada a la investigación y a la docencia”. Esta condición exige 
también el desarrollo de habilidades pedagógicas para la integración de las TIC en el proceso de la investigación formativa en el pregrado y en el posgrado.

Frente a esta situación es importante, mencionar que las universidades en el Perú tienen el objetivo de promover la integración de las tecnologías de la información y comunicación (TIC) en todos los cursos, programas y modalidades de enseñanza. Sin embargo, se puede notar el poco interés por parte de los docentes, por desarrollar habilidades que les permita familiarizarse con los contenidos digitales, logrando así el desarrollo de competencias digitales. Por lo cual, la investigación radica en la identificación de las competencias digitales que poseen los estudiantes en el transcurso de su quehacer académico y cuánto han desarrollado dichas habilidades en épocas de clases $100 \%$ virtuales debido a la pandemia de la Covid-19.

Un posible pronóstico a esta realidad sería que el estudiante que carece de conocimientos sobre el entorno virtual puede tener un insuficiente desempeño bajo esta modalidad, generando cierta frustración a futuro. En este contexto, los docentes se deben capacitar para desarrollar-clases virtuales, desarrollando un modelo didáctico adaptado a esta realidad, con foco en los estudiantes y centrando en el desarrollo de sus competencias y principales necesidades (Pascual, Ortega, Ferra y Fombona, 2019). Por ello, es conveniente realizar un diagnóstico de cada una de las capacidades digitales que poseen los educandos en tiempos de covid-19.

\section{MATERIALES Y MÉTODOS}

El presente estudio parte de un enfoque cuantitativo - analítico basado en un análisis descriptivo, en donde se integraron métodos y técnicas de recolección y análisis de información basados en la medición numérica. El diseño fue no experimental que consistió en identificar mediante un cuestionario, la percepción de los estudiantes universitarios con respecto al desarrollo de las competencias digitales durante clases.

La investigación se desarrolló en la Universidad Tecnológica del Perú sede Chiclayo, Perú, en el marco de la asignatura Comprensión y Redacción de textos en la modalidad virtual, en donde los estudiantes acceden a través de la plataforma virtual Zoom. La población estuvo conformada por 112 estudiantes del primer ciclo de la facultad de Humanidades. $75 \%$ de los participantes fueron hombres y el $25 \%$ mujeres, con rangos de edad entre los 17 y 18 años edad. 
Para diagnosticar el desarrollo de las competencias digitales, se aplicó un cuestionario, el cual incluyó preguntas sobre el uso de los dispositivos electrónicos y su nivel de conocimiento sobre este tema. El cuestionario estuvo compuesto por 15 preguntas, el cual se aplicó a través de una encuesta creada en Google drive. El instrumento estuvo dividido en cinco dimensiones: información, comunicación, creación, seguridad y resolución.

Para el análisis y codificación de los datos se utilizó el programa estadístico SPSS versión 25 , en donde se determinó que el instrumento era confiable (0.802) según la consistencia interna (Alfa de Cronbach) de los ítems. El cuestionario fue validado por tres expertos de la Maestría en Tecnología de la Educación, con experiencia en el manejo de las TIC en educación superior.

Posteriormente se realizó una revisión bibliográfica de artículos, estudios, encuestas, monografías y otros documentos sobre el tema de estudio planteado. Para ello utilizamos las bases documentales existentes (Dialnet, Scielo, Scopus, Web of Science y Google académico), lo que nos ha permitido delimitar el marco conceptual o teórico en el que se sustenta el artículo. En la revisión de la literatura y su selección se utilizó gestores bibliográficos (Mendeley - Zotero) que facilita el manejo adecuado de las referencias y su clasificación.

\section{RESULTADOS Y DISCUSIÓN}

La presentación y análisis de los resultados se muestran en torno al cuestionario de competencias digitales aplicado a los estudiantes del 1 semestre académico de una universidad privada de Chiclayo. De modo que la información se organizó en tablas y figuras, con los hallazgos más resaltantes del estudio.

Tabla 1 Acceso a dispositivos e internet durante las clases virtuales

\begin{tabular}{llc}
\hline Dimensión & Unidad de medida & \% estudiantes \\
\hline \multirow{2}{*}{ Dispositivo de acceso } & Laptop & $57 \%$ \\
& Tablet & $3 \%$ \\
& Computadora & $32 \%$ \\
Tiempo de conexión en & Smartphone & $8 \%$ \\
internet & Cinco a más horas al día & $81,4 \%$ \\
& Tres a más a horas al día & $16 \%$ \\
Temas de búsqueda & Una hora diaria & $2,6 \%$ \\
& Académicos & $37 \%$ \\
& Investigación & $11 \%$ \\
& Noticias de actualidad & $8 \%$ \\
& Redes Sociales & $44 \%$ \\
\hline
\end{tabular}


Nota. El análisis de los resultados nos presenta tres apartados. En el primero se describen los dispositivos de acceso a internet de los estudiantes. La segunda parte establece el tiempo de conexión en internet y por el último los de temas de búsqueda recurrentes por parte de los estudiantes.

El resultado indicó un alto de porcentaje de estudiantes que acceden a sus clases a través de una laptop (57\%). La cantidad de horas que permanecen conectados a internet es desigual, ya que la mayoría permanece más de cinco horas al día $(81,4 \%)$ y otros entre tres a más horas al día (16\%). El $44 \%$ de alumnos permanece en Internet para revisar las redes sociales, y el $37 \%$ para temas académicos (tabla 1). Los resultados revelan lo indicado por Levano, et. Al (2019) quienes mencionan que se ha evidenciado un déficit en las herramientas digitales por parte de los estudiantes. A pesar que más de la mitad de ellos, pasa de más de cinco horas diarias conectados a la red ya se a través de la PC, computador portátil, Tablet o smartphones de última generación, no son capaces de extraer un mayor aprovechamiento de dichas plataformas y por ende se percibe cierta obsolescencia de competencias digitales, ya que utilizan la tecnología para jugar, interactuar en redes sociales y realizar otras actividades alejadas del proceso educativo.

Tabla 2 Niveles de la variable Competencia digitales

\begin{tabular}{|c|c|c|c|c|c|c|c|c|c|c|}
\hline \multirow{3}{*}{ Niveles } & \multicolumn{10}{|c|}{ Competencias digitales } \\
\hline & \multicolumn{2}{|c|}{ Información } & \multicolumn{2}{|c|}{ Comunicación } & \multicolumn{2}{|c|}{ Creación } & \multicolumn{2}{|c|}{ Seguridad } & \multicolumn{2}{|c|}{ Resolución } \\
\hline & $\mathrm{f}$ & $\%$ & f & $\%$ & $\mathrm{f}$ & $\%$ & $\mathrm{f}$ & $\%$ & $\mathrm{f}$ & $\%$ \\
\hline Avanzado & 47 & $42,5 \%$ & 41 & $35,7 \%$ & 31 & $30,1 \%$ & 62 & $59,5 \%$ & 53 & $50,9 \%$ \\
\hline Intermedio & 19 & $14 \%$ & 65 & $62,3 \%$ & 72 & $59,9 \%$ & 44 & $30,5 \%$ & 47 & $33,1 \%$ \\
\hline Básico & 40 & $37,5 \%$ & 0 & $0,0 \%$ & 0 & $0,0 \%$ & 0 & $0,0 \%$ & 3 & $2,1 \%$ \\
\hline \multirow[t]{2}{*}{ Total } & 10 & $6 \%$ & 10 & $2 \%$ & 10 & $10 \%$ & 10 & $10 \%$ & 10 & $6 \%$ \\
\hline & 6 & $\%$ & 6 & $\%$ & 6 & $\%$ & 6 & $\%$ & 6 & $\%$ \\
\hline
\end{tabular}

$\overline{\text { Nota. } \text { El análisis de los resultados nos presenta los niveles de la variable Competencias }}$ digitales, mediante tres niveles: avanzado, intermedio y básico.

El procesamiento de información de los datos recogidos de la encuesta en los estudiantes de una universidad Tecnológica del Perú para la variable competencias digitales indicó que el 38,7\% se encuentran en el nivel avanzado y un 61,3\% están en el nivel intermedio; en la dimensión información el 42,5\% se encuentran en el nivel avanzado y el 14\% en el nivel intermedio y en el nivel básico un 37,5\%; en la dimensión Comunicación el 35,7\% 
se encuentran en el nivel avanzado, el 62,3\% se ubica en el nivel intermedio; en la dimensión Creación el 30,1\% se encuentran en el nivel avanzado, el 59,9\% se ubica en el nivel intermedio; en la dimensión Seguridad el 59,5\% se encuentran en el nivel avanzado y un 30,5\% está el nivel intermedio; en la dimensión resolución el 50,9\% se encuentran en el nivel avanzado, un 33,1\% está el nivel intermedio y un $6 \%$ se encuentra en el nivel básico (Tabla 2). Es necesario poner de manifiesto lo indicado por Padilla, Gámiz y Romero (2019) quienes aseveran que la competencia digital es el conjunto de conocimientos, habilidades, actitudes, estrategias y concienciación que el uso de las TIC y de los medios digitales requiere para realizar tareas, resolver problemas, comunicar, gestionar la información, colaborar, crear y compartir contenidos y generar conocimiento de forma efectiva, eficaz, adecuada, crítica, creativa, autónoma, flexible, ética, reflexiva para el trabajo, el ocio, la participación, el aprendizaje, la socialización, el consumo y el empoderamiento. Entre las principales competencias digitales tenemos: la alfabetización en información y datos, la comunicación, la creación de contenidos digitales, seguridad y resolución de problemas.

Figura 1. Acciones que realizan los estudiantes ante cualquier duda, durante las clases virtuales en la plataforma virtual Zoom.

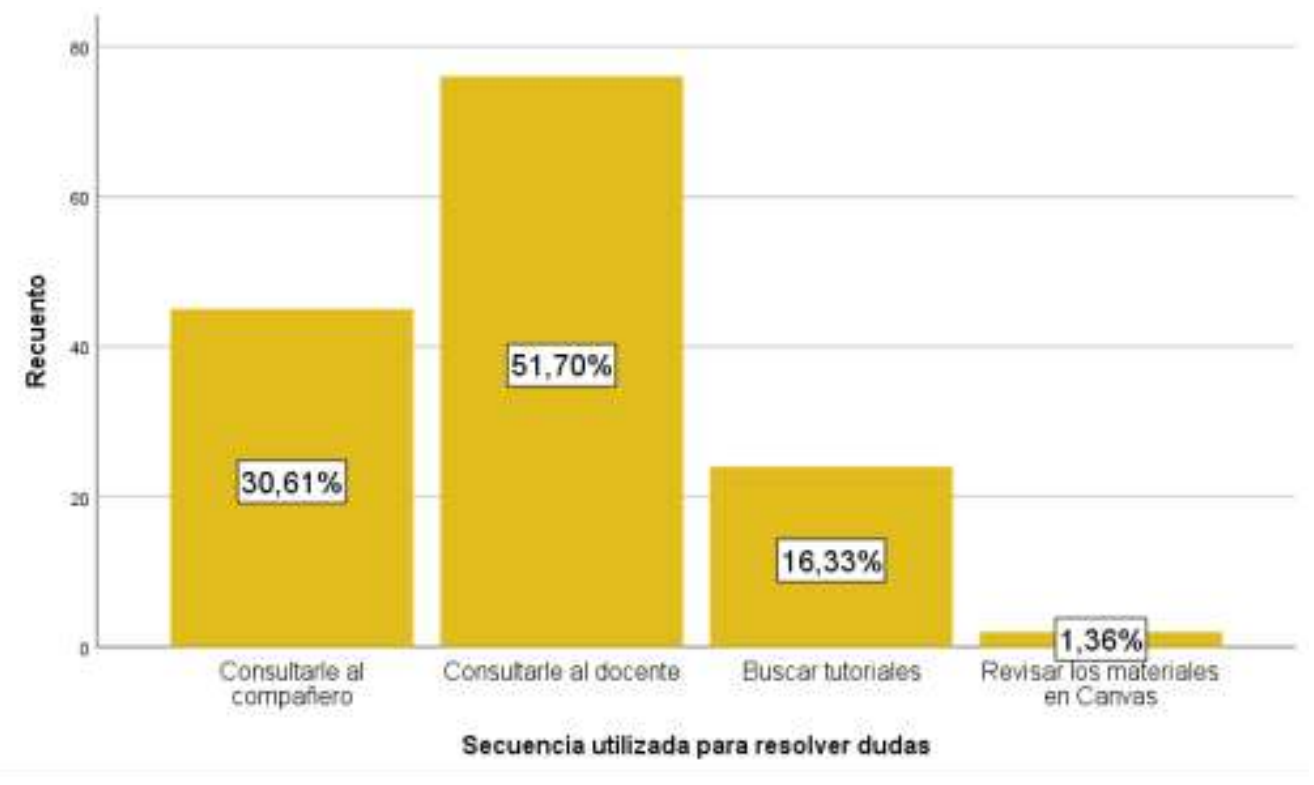

Nota. La figura 1 nos muestra como la consulta al docente es la acción más recurrente de los estudiantes ante cualquier duda en las clases virtuales $(51,70 \%)$, seguido de la consulta a sus compañeros $(30,61 \%)$. Sin embargo, solo el 1,36\% hacen uso de los recursos tecnológicos que les brinda la plataforma Canvas. 


\section{CONCLUSIONES}

La situación actual por covid-19 ha generado que los estudiantes del nivel superior realicen sus clases de manera remota. Por lo cual, desarrollar competencias digitales es uno de los retos más importantes de la educación superior. Si bien es cierto, que los estudiantes son parte de la era digital y dominan herramientas de información, requieren un mejor uso de las redes e información disponible de internet como elemento para el desarrollo de habilidades digitales que contribuyan con su formación profesional y laboral.

Este desafió es una responsabilidad compartida en la que, los docentes de Educación Superior deben capacitarse y conocer de cerca los planes y programas informáticos para poder aplicar las TIC en sus clases virtuales, como bien apuntan muchos autores, muchos docentes no se familiarizan con la tecnología, porque quizá se educaron en otra época, sin embargo, el reto es adoptar las competencias digitales y facilitar las herramientas a los estudiantes en el proceso de enseñanza - aprendizaje.

Finalmente, los resultados arrojan resultados no tan alentadores relacionados con el desarrollo de las competencias digitales, siendo esta el principal problema que afronta la Educación Superior en la actualidad, debido a que los estudiantes utilizan la tecnología para actividades que no aportan en nada con su educación y para lo cual se debe cambiar el panorama e implementar estrategias que les permitan motivarse y hacer uso de las herramientas tecnológicas para su formación académica.

\section{LISTA DE REFERENCIAS}

Aguirre, G., y Ruiz, M. (2012). Competencias digitales y docencia: una experiencia desde la práctica universitaria. Innovus, 12(59), 165-267. Obtenido de http://www.scielo.org.mx/pdf/ie/v12n59/v12n59a9.pdf

Arias, M., Torres, T., y Yañez, J. (2014). El desarrollo de competencias digitales en la educación superior. Historia y Comunicación Social, 19, 355-366.

Cabero, J., y Llorente, M. (2008). La alfabetización digital de los alumnos: competencias digitales para el siglo XX. Revistas Portuguesa de Pedagogía, 42(2), 18-21.

Caraballo, A., Domínguez, M., y Paralela, C. (2011). El entorno virtual: Un espacio para el aprendizaje colaborativo. Revista Electrónica de Tecnología Educativa, 11(35), 125-128. 
Chávez, F. H., Cantú, M., y Rodríguez, C. M. (2016). Competencias digitales y tratamiento de información desde la mirada infantil. Revista Electrónica de Investigación Educativa, 18(1), 209-220. Obtenido de http://www.scielo.org.mx/pdf/redie/v18n1/v18n1a15.pdf

Cobos, J. C., Jaramillo, L., y Vinueza, S. (2019). Las competencias digitales en docentes y futuros profesionales de la Universidad Central del Ecuador. Cátedra, 2(1), 7697. doi:https://doi.org/10.29166/catedra.v2i1.1560

Córdova, A., y Rossani, G. (2020). COVID-19 revisión de la literatura y su impacto en la realidad sanitaria peruana. Revista de la Facultad de Medicina Humana, 20(3), 17.

Levano, L., Sanchez, S., Guillén, P., Tello, S., Herrera, N., y Collantes, Z. (2019). Competencias digitales y educación. Propósitos y Representaciones, 7(2), 25-45.

Maguiña, C., Acosta, R. G., y Tequen, A. (2020). El nuevo Coronavirus y la pandemia del Covid-19. Rev Med Hered, 31(2).

Mendoza, L. (2020). Lo que la pandemia nos enseñó sobre la educación a distancia. Revista Latinoamericana de Estudios Educativos, L.

Murillo, F. J., y Duk, C. (2020). El Covid-19 y las Brechas Educativas. Revista latinoamericana de educación inclusiva, 14(1), 15-26. Obtenido de https://scielo.conicyt.cl/scielo.php?script=sci_arttext\&pid=S071873782020000100011

Padilla, A. L., Gámiz, V., y Romero, M. (2019). Competencia digital docente: apuntes sobre su conceptualización. Revista de Cultura Digital, 7(2), 125-135.

Pascual, MA., Ortega, J., Perez, M., y Fombona, J. (2019). Competencias Digitales en los Estudiantes del Grado de Maestro de Educación Primaria. El caso de tres Universidades Españolas. Formación universitaria, 12(6), 141-150.

Rojas, V. R., Zeta, A., y Jiménez, R. (2020). Competencias digitales en una universidad pública peruana. Revista Conrado, 16(77), 125-130.

Silas, J. C., y Vázquez, S. (2020). El docente universitario frente a las tensiones que le plantea la pandemia. Revista Latinoamericana de Estudios Educativos, L. 